\title{
A Probabilistic Approach to Capsize of Ship in Random Astern Seas
}

\author{
by Masami Hamamoto*, Member \\ James P. Panjaitan**, Student Member \\ Abdul Munif**, Student Member
}

\begin{abstract}
Summary
The purpose of this paper is to investigate the insight of capsizing modes in details and the probability of capsize in a random sea by making use of a mathematical model for simulations. Firstly a prediction method to calculate critical wave height leading to capsize are proposed for three modes of parametric resonance, harmonic resonance and pure loss of stability. Secondly according to Belenky's ${ }^{4,5}$ simplified method to compute the probability of capsizing, the probabilities of capsizing caused by parametric resonance and harmonic resonance are evaluated for a ship running with the specified combination of heading angle and ship speed in random waves. Finally these computed outcomes are discussed for the 15000 GT container carrier.
\end{abstract}

\section{Introduction}

Stability against capsizing in severe seas is one of the most fundamental requirement considered by the naval architect when designing a ship. For this requirement, it is expected to establish probabilistic stability assessment for the random response of the ship to a random sea specified by wave spectrum. Recently several experimental ${ }^{11,12,13,14,15)}$, and analytical studies have been conducted for this purpose in the related countries. According to these recent developments, this paper attempts to make a practical method to compute critical rolling leading to capsize and the probability of capsizing in a random sea.

There are four principal modes which are called parametric resonance, harmonic resonance, pure loss of stability and broaching to $^{10)}$. Three modes without broaching-to may occur in waves of the length nearly equal to ship length but in different encounter frequencies. The experimental observation suggests that probability of capsize may be related to the probability of the ship encountering a wave group having the characteristics necessary to cause capsize. If a ship is running through a regular following sea, the ship will experience periodic variations in its transverse stability and these variations will affect the roll motion of the ship $^{6)}$. An approximately similar phenomenon might be expected if the ship encounters a wave group having sufficient regularity and steepness in random astern seas.

* Osaka University

** Graduate School, Osaka University

Received 10th July 1997

Read at the Autumn meeting 14, 15th Nov. 1997
When a ship is running with a constant speed $U$ and heading angle $\chi$ in regular astern waves $^{1,2,3)}$ of the frequency $\omega$, the encounter frequency $\omega_{e}$ is given by

$$
\omega_{e}=\omega-\frac{\omega^{2}}{g} U \cos \chi
$$

or the nondimensional form $\omega_{e} \sqrt{L / g}$ is

$$
\omega_{e} \sqrt{L / g}=\sqrt{2 \pi L / \lambda}\left[1-\sqrt{2 \pi L / \lambda} F_{n} \cos \chi\right]
$$

where $g$ is the gravitational acceleration, $L$ ship length, $\lambda$ wave length and $F_{n}$ Froude-number.

Fig. 1 shows the relationship between $\omega_{e} \sqrt{L / g}$ and $F_{n}$ $\cos \chi$ for $\lambda / L=1$. If the natural rolling frequency $\omega_{\phi}$ is given, from this figure it will be possible to find the combination of Froude number $F_{n}$ and heading angle $\chi$ which may cause the parametric resonance, harmonic resonance and pure loss of stability. That is to say, the parametric resonance takes place when the natural

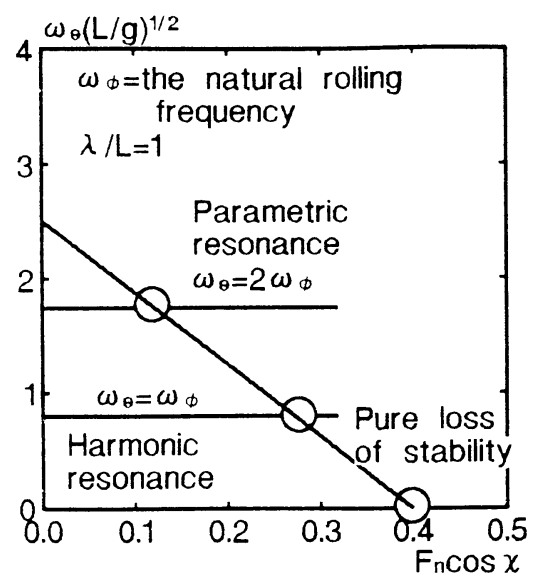

Fig. 1 The encounter frequency vs $\mathrm{F}_{\mathrm{n}} \cos \chi$ 
rolling frequency $\omega_{\phi}$ is nearly equal to one-half encounter frequency $\omega_{e}$ in relative low speed and small heading angle. The harmonic resonance occurs when the natural rolling frequency $\omega_{\phi}$ is nearly equal to encounter frequency $\omega_{e}$ and the pure loss of stability occurs at high speed nearly equal to the wave phase velocity so that the ship remains almost stationary relative to the crest for sufficient length of time to capsize. The problem here is to investigate a critical wave height $H_{c}$ and metacentric height $G M_{c}$ which lead to capsize of ship running in astern seas.

In order to achieve this, free running model experiments carried out for a $15000 \mathrm{GT}$ container carrier and 135 GT purse seiner under panel RR 71 of Shipbuilding Research Association of Japan and the papers about the outline of experimental results have been already published $^{9,10)}$.

The purpose of present paper is to investigate the insight of capsizing modes in details and the probability of capsize in a random sea by making use of a mathematical model for simulations.

\section{Mathematical Model for Numerical Simulations}

In general ship motions leading to capsize in severe astern seas is nonlinear and complicated phenomena up to six degrees of freedom ${ }^{10)}$. However, the essential features of the phenomenon come from the variation of righting arm dependent on the longitudinal position of ship to waves ${ }^{7}$. In this situation the righting arm varies with respect to not only the rolling angle but also heave and pitch angle in the exact position of ship to waves. Furthermore, the heave and pitch angle in heeled condition are mainly determined in static balance relating to the instantaneous submerged hull varying with respect to the relative position of ship to waves. According to this essential features, the rolling angle $\phi(t)$ of ship in astern seas has to be described by combination of roll, heave and pitch motions which leads to the following equations

$$
\begin{aligned}
\left(I_{x}+J_{x}\right) \ddot{\phi}+K_{\dot{\phi}} \dot{\phi}+W G Z(\phi, t) & =K(t) \\
\rho g \int_{L} A(x, t) d x+Z(t) & =m g \\
\rho g \int_{L} x A(x, t)+M(t) & =0
\end{aligned}
$$

where $I_{x}+J_{x}$ is the moment and added moment of inertia, $K_{\phi}$ the roll damping coefficient, $W$ ship weight, $G Z(\phi, t)$ the time dependent righting arm, $Z(t), K(t)$ and $M(t)$ are the heaving force, rolling and pitching moments acting on the instantaneous submerged hull, $A(x, t)$ the instantaneous submerged sectional area which is also a function of heave $\zeta_{G}$ and pitch $\theta$, as shown in Fig. 2, $m$ the ship mass, $g$ the gravity acceleration and $\rho$ fluid density.

On the other hand the sea surface elevation $\zeta_{w}(t, x, y)$ is given by the sum sinusoidal waves in the body coordinate system $G-x, y, z$ as

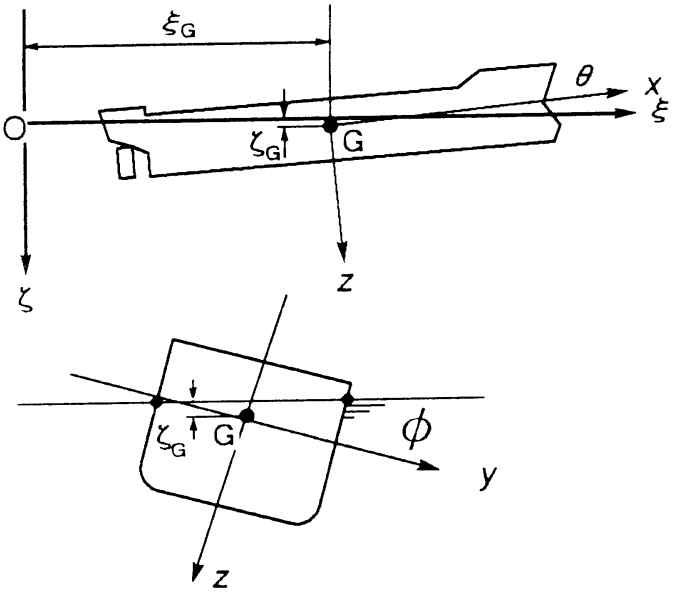

Fig. 2 Co-ordinate systems

$$
\begin{aligned}
\zeta_{w} & =-\zeta_{G}+x \theta+\sum_{n=1}^{N} C_{n} \cos \left[\frac { \omega _ { n } ^ { 2 } } { g } \left(\xi_{G}+x \cos \chi\right.\right. \\
& -y \sin \chi \cos \phi+z \sin \chi \sin \phi) \\
& \left.-\left(\omega_{n}-\frac{\omega_{n}^{2}}{g} U \cos \chi\right) t+\epsilon_{n}\right]
\end{aligned}
$$

where $N$ is the number of component wave, $\omega_{n}$ the circular frequency of $n$-th wave, $\epsilon_{n}$ random phase angle, $\chi$ the heading angle of ship to waves, $\xi_{G}$ the initial position of ship running with speed $U, C_{n}$ the amplitude of the $n-t h$ wave given by the power spectrum $S(\omega)$ as $C_{n}=\sqrt{2 S\left(\omega_{n}\right) \Delta \omega}$ and power spectrum $S(\omega)$ is given by ITTC 1978 in the following form

$$
S(\omega)=\frac{172.8}{\omega^{5}}\left(\frac{H_{1 / 3}^{2}}{T_{01}^{4}}\right) \exp \left[-\frac{691}{\omega^{4} T_{01}^{4}}\right]
$$

The wave pressure $p(t, x, y, z)$ is

$$
\begin{aligned}
p= & \rho g\left(\zeta_{G}-x \theta+y \sin \phi+z \cos \phi\right) \\
& -\rho g \sum_{n=1}^{N} C_{n} \exp \left[-\frac{\omega_{n}^{2}}{g} d\right] \cos \left[\frac { \omega _ { n } ^ { 2 } } { g } \left(\xi_{G}\right.\right. \\
& +x \cos \chi-y \sin \chi \cos \phi+z \sin \chi \sin \phi) \\
& \left.-\left(\omega_{n}-\frac{\omega_{n}^{2}}{g} U \cos \chi\right) t+\epsilon_{n}\right]
\end{aligned}
$$

The Froude-Krylov force $Z(t)$ and moments $K(t)$ and $M(t)$ can be obtained by integrating the pressure over the entire submerged surface of the ship. After some mathematical manipulations in details, the equations of motion can be rewritten in the following form

$$
\begin{aligned}
& \ddot{\phi}+2 \alpha_{e} \dot{\phi}+\omega_{\phi}^{2}\left[\frac{G Z(\phi, t)}{G M}\right]=\omega_{\phi}^{2} \sum_{n=1}^{N}\left[A _ { n } \operatorname { s i n } \left(\omega_{n}\right.\right. \\
& \left.\quad-\frac{\omega_{n}^{2}}{g} U \cos \chi\right) t \\
& \left.\quad-B_{n} \cos \left(\omega_{n}-\frac{\omega_{n}^{2}}{g} U \cos \chi\right) t\right] \sin \chi \\
& \rho g \int_{L} A(x) d x+\rho g \sum_{n=1}^{N}\left[H_{n} \cos \left(\omega_{n}-\frac{\omega_{n}^{2}}{g} U \cos \chi\right) t\right. \\
& \left.\quad+I_{n} \sin \left(\omega_{n}-\frac{\omega_{n}^{2}}{g} U \cos \chi\right) t\right]=m g \\
& \rho g \int_{L} x A(x) d x+\rho g \sum_{n=1}^{N}\left[J_{n} \cos \left(\omega_{n}-\frac{\omega_{n}^{2}}{g} U \cos \chi\right) t\right.
\end{aligned}
$$




$$
\left.+K_{n} \sin \left(\omega_{n}-\frac{\omega_{n}^{2}}{g} U \cos \chi\right) t\right]=0
$$

where $G Z(\phi, t), F_{n}(x), A_{n}, B_{n}, H_{n}, I_{n}, J_{n}$ and $K_{n}$ are

$$
\begin{aligned}
& G Z(\phi, t)=\rho g \int_{L}\left[y_{B}(x) \cos \phi-z_{B}(x) \sin \phi\right] A(x) d x \\
& F_{n}(x)=C_{n} \frac{\omega_{n}^{2}}{g} \exp \left[-\frac{\omega_{n}^{2}}{g} d\right] \frac{\sin \left[\frac{\omega_{n}^{2}}{g} B(x) \sin \chi\right]}{\frac{\omega_{n}^{2}}{g} B(x) \sin \chi}
\end{aligned}
$$$$
A_{n}=\frac{\rho g}{W G M} \int_{L}\left[z_{B}(x) \cos \phi+y_{B}(x) \sin \phi\right]
$$$$
\times A(x) F_{n}(x) \cos \left[\frac{\omega_{n}^{2}}{g}\left(\xi_{0}+x \cos \chi\right)+\epsilon_{n}\right] d x
$$$$
B_{n}=\frac{\rho g}{W G M} \int_{L}\left[z_{B}(x) \cos \phi+y_{B}(x) \sin \phi\right]
$$$$
\times A(x) F_{n}(x) \sin \left[\frac{\omega_{n}^{2}}{g}\left(\xi_{0}+x \cos \chi\right)+\epsilon_{n}\right] d x
$$$$
H_{n}=\int_{L} A(x) F_{n}(x) \cos \left[\frac{\omega_{n}^{2}}{g}\left(\xi_{0}+x \cos \chi\right)+\epsilon_{n}\right] d x
$$$$
I_{n}=\int_{L} A(x) F_{n}(x) \sin \left[\frac{\omega_{n}^{2}}{g}\left(\xi_{0}+x \cos \chi\right)+\epsilon_{n}\right] d x
$$

$$
\begin{aligned}
& J_{n}=\int_{L} x A(x) F_{n}(x) \cos \left[\frac{\omega_{n}^{2}}{g}\left(\xi_{0}+x \cos \chi\right)+\epsilon_{n}\right] d x \\
& K_{n}=\int_{L} x A(x) F_{n}(x) \sin \left[\frac{\omega_{n}^{2}}{g}\left(\xi_{0}+x \cos \chi\right)+\epsilon_{n}\right] d x
\end{aligned}
$$

and $G M$ metacentric height, $\omega_{\phi}$ natural roll frequency, $y_{B}(x)$ and $z_{B}(x)$ are the center of buoyancy of submerged sectional area $A(x)$.

\section{Computation for Probability of Capsizing}

It may be possible to carried out numerical simulation for critical motions and capsize of the ship running in a random astern sea. But it would be impossible to find a reasonable criterion for stability without making use of probabilistic approaches because of random processes of both waves and ship motions. Although several concepts were made for evaluating a probability of ship capsizing, recently Belenky ${ }^{4,5)}$ in 1993 and 1994 has proposed a practical and interesting method to estimate the probability of capsize in random seas. His method was derived from a concept that the rolling angle of ship in critical condition is mainly affected by free rolling in a transient state. According to his method, the first equation in Eq. ( 7 ) is linearized near the vanishing stability of righting arm curve GZ as follows

$$
\ddot{\phi}+2 \alpha_{e} \dot{\phi}+\omega_{\eta}^{2}\left(\phi_{V}-\phi\right)=f(t)
$$

So that the equation of free rolling in the small range $\Delta$ $\phi=\phi-\phi_{V}$ as shown in Fig. 3 can be linearized as

$$
\Delta \ddot{\phi}+2 \alpha_{e} \Delta \dot{\phi}-\omega_{v}^{2} \Delta \phi=0
$$

then the solution of $\phi(t)$ is

$$
\begin{aligned}
& \phi(t)=A e^{\lambda_{1} t}+B e^{-\lambda_{2} t}+\phi_{V} \\
& A=\frac{\lambda_{2}\left(\phi_{0}-\phi_{V}\right)+\dot{\phi}_{0}}{\lambda_{1}+\lambda_{2}} \quad B=\frac{\lambda_{2}\left(\phi_{0}-\phi_{V}\right)-\dot{\phi}_{0}}{\lambda_{1}+\lambda_{2}} \\
& \lambda_{1}=\sqrt{\alpha_{e}^{2}+\omega_{V}^{2}}-\alpha_{e} \quad \lambda_{2}=\sqrt{\alpha_{e}^{2}+\omega_{V}^{2}}+\alpha_{e} \\
& \omega_{V}^{2}=\frac{W}{I_{x}+J_{x}}\left(\frac{d G Z}{d \phi}\right)_{\phi=\phi V}
\end{aligned}
$$

where $f(t)$ is the wave excitation, $\phi_{0}$ and $\dot{\phi}_{0}$ are the initial values at the local time in the range $\Delta \phi$.

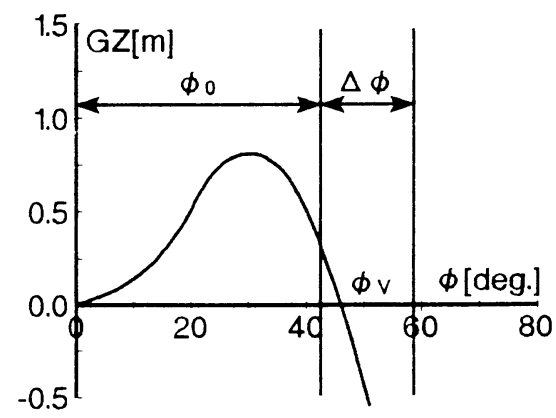

Fig. 3 A small range $\Delta \phi$ near vanishing stability

The first term in Eq. (12) develops the rolling into an unstable behavior when the coefficient $A>0$ because $\lambda_{1}$ is always positive. Thus if the coefficient $A>0$ for positive rolling angle, then capsizing will take place and if the coefficient $A<0$ then capsizing will not take place because the rolling swings back to the opposite direction. Consequently it is pointed out that the sign of the coefficient $A$ is a criterion of the critical rolling. In the present paper $\phi(t)$ and $\dot{\phi}(t)$ can be given by the numerical solution of Eq. ( 7 ) with nonlinear GZ curve. The influence of wave excitation is small in the capsize process because the ship loses sensitivity to wave excitation when the rolling angle is bigger than the angle at the maximum of $G Z$ curve. Regarding to this fact, it can be said that the influence of the wave excitation is given only by $\dot{\phi}$ in the range $\Delta \phi$ as

$$
\dot{\phi}_{0}=\left[A-\frac{\lambda_{2}\left(\phi_{0}-\phi_{V}\right)}{\lambda_{1}+\lambda_{2}}\right]\left(\lambda_{1}+\lambda_{2}\right)
$$

Then the probability density function of coefficient $A$ is given by

$$
\begin{aligned}
f(A) & =\frac{1}{\sqrt{2 \pi}\left[\sigma_{\dot{\phi}} /\left(\lambda_{1}+\lambda_{2}\right)\right]} \\
& \times \exp \left[-\frac{1}{2} \frac{\left(A-\lambda_{2}\left(\phi_{0}-\phi_{V}\right) /\left(\lambda_{1}+\lambda_{2}\right)\right)^{2}}{\sigma_{\dot{\phi}}^{2} /\left(\lambda_{1}+\lambda_{2}\right)^{2}}\right]
\end{aligned}
$$

here the coefficient $A$ is positive for positive rolling angle and negative for negative rolling angle, therefore the probability distribution of the coefficient $A$ for both sides of rolling angle is

$$
\begin{aligned}
P(A>0) & =2 \int_{0}^{\infty} f(A) d A \\
& =1+\operatorname{erf}\left[\frac{\lambda_{2}\left(\phi_{0}-\phi_{V}\right)}{\sqrt{2} \sigma_{\dot{\phi}}}\right]
\end{aligned}
$$

where $\operatorname{erf}(x)$ is the error function, and $\operatorname{erf}(-x)=-\operatorname{erf}(x)$.

One more important index is pointed out for the probability of at least one up-crossing of level $\phi_{0}$ during time $T$ in which $P\left(\phi>\phi_{0}\right)$ can be calculated according to the exponential law of Poisson distribution

$$
\begin{aligned}
& P\left(\phi>\phi_{0}\right)=1-e^{-E(\phi 0) T} \\
& E\left(\phi_{0}\right)=\frac{1}{2} \int_{-\infty}^{\infty}|\dot{\phi}| f\left(\phi_{0}, \dot{\phi}\right) d \dot{\phi} \\
& \quad=\frac{1}{2 \pi}\left(\frac{\sigma_{\dot{\phi}}}{\sigma_{\phi}}\right) \exp \left(-\frac{\phi_{0}^{2}}{2 \sigma_{\phi}^{2}}\right)
\end{aligned}
$$




$$
f(\phi, \dot{\phi})=\frac{1}{2 \pi \sigma_{\phi} \sigma_{\dot{\phi}}} \exp \left[-\frac{1}{2}\left(\frac{\phi^{2}}{\sigma_{\phi}^{2}}+\frac{\dot{\phi}^{2}}{\sigma_{\dot{\phi}}^{2}}\right)\right]
$$

where $\sigma_{\phi}^{2}$ and $\sigma_{\dot{\phi}}^{2}$ are variances of rolling angle and angular velocity, $E\left(\phi_{0}\right)$ the number of up-crossing level $\phi_{0}$ and $f(\phi, \dot{\phi})$ joint probability density function for $\phi$ and $\dot{\phi}$.

Finally, a probability of capsizing during time $T$ can be calculated in the following form

$$
P(T)=2 P\left(\phi>\phi_{0}\right) \cdot P(A>0)
$$

That is the outline of a simplified method proposed by Belenky4) in 1993.

\section{Examples of Numerical Simulation}

Several examples of critical modes simulated by numerical computations are presented here. These computations were performed for a $15000 \mathrm{GT}$ container carrier for which free running model experiments were previously carried out in seakeeping basin of NRIFE and SRI. Detail of experiments with this model are given in the paper by Umeda, et $\mathrm{al}^{9)}$ and Hamamoto, et $\mathrm{al}^{10)}$. The principal dimensions of ship and the model are given in Table 1 . The righting arm curves $G Z$ in still water, at wave crest and trough amidships for $G M$ $=0.318 \mathrm{~m}$, and $0.6 \mathrm{~m}$ are shown in Fig. 4 . Time domain simulations were carried out at ship scale for the critical modes leading to capsize in both regular and random waves.

Critical wave height leading to capsize in a regular $\underline{\text { wave }}$

Firstly the parametric resonances at $\omega_{e}=2 \omega_{\phi}$ are simulated for the ship running at $F_{n}=0.195$ of $\mathrm{GM}=$ $0.318 \mathrm{~m}$ and $\mathrm{Fn}=0.109$ of $\mathrm{GM}=0.6 \mathrm{~m}$ and heading angle $\chi=0$ in regular waves of $\lambda / L=1$. The time histories of roll and pitch in waves of the height near critical modes leading to capsize are shown in Fig. 5. The initial

Table 1 Principal Dimension of Container Carrier

\begin{tabular}{|l|l|c|r|}
\hline \hline \multicolumn{2}{|c|}{ Items } & Ship & Model \\
\hline Length & $\mathrm{L}(\mathrm{m})$ & 150.0 & 2.5 \\
Breadth & $\mathrm{B}(\mathrm{m})$ & 27.2 & 0.453 \\
Depth & $\mathrm{D}(\mathrm{m})$ & 13.5 & 0.225 \\
Draft & $d_{f}(\mathrm{~m})$ & 8.50 & 0.142 \\
& $d_{a}(\mathrm{~m})$ & 8.50 & 0.142 \\
Block Coef. & $C_{b}$ & 0.667 & 0.667 \\
Metacentric & $\mathrm{GM}(\mathrm{m})$ & 0.318 & 0.0053 \\
height & & 0.60 & 0.077 \\
Natural roll & $T_{\phi}(\mathrm{sec})$ & 38.73 & 5.0 \\
period & & 27.01 & 3.5 \\
Model Scale & - & - & $1 / 60$ \\
\hline \hline
\end{tabular}
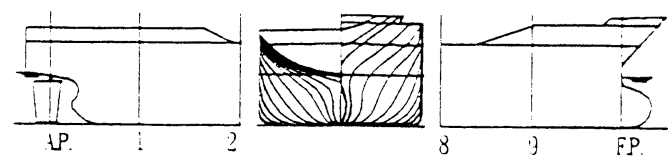
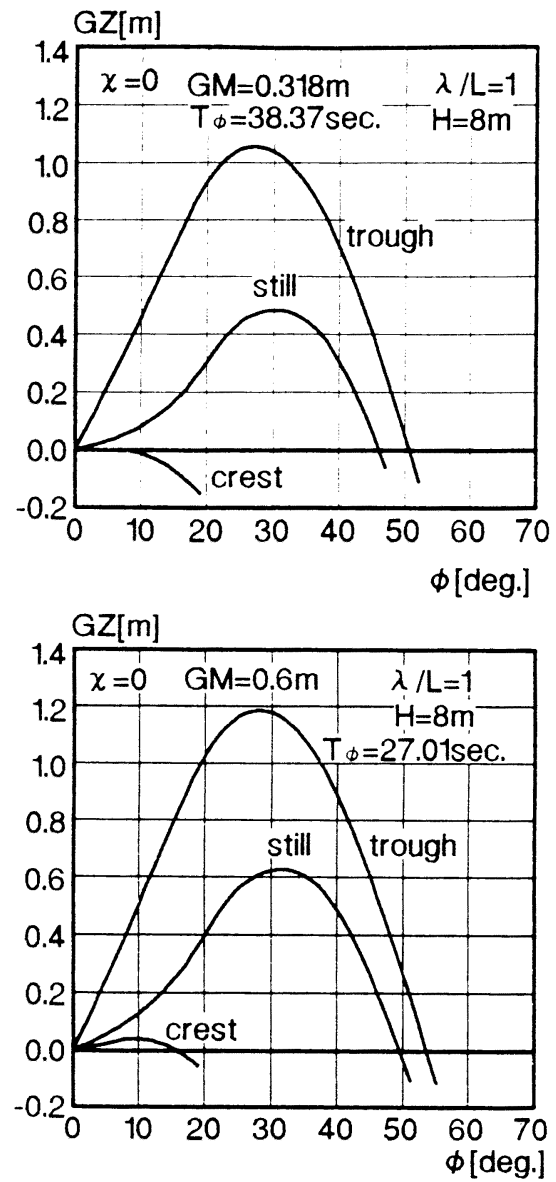

Fig. 4 Righting lever curves GZ in still, wave trough and crest amidship for $\mathrm{GM}=0.318 \mathrm{~m}$, and $\mathrm{GM}=$ $0.6 \mathrm{~m}$

$\mathrm{GM}=0.318 \mathrm{~m}, \mathrm{Fn}=0.195, \mathrm{H}=7.1 \mathrm{~m}, \chi=0$

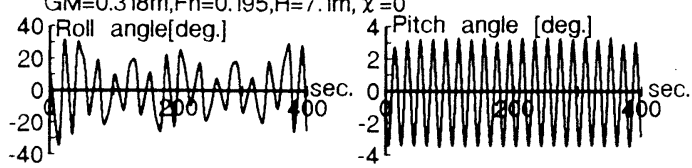

$\mathrm{GM}=0.6 \mathrm{~m}, \mathrm{Fn}=0.109, \mathrm{H}=10 \mathrm{~m}, \chi=0$

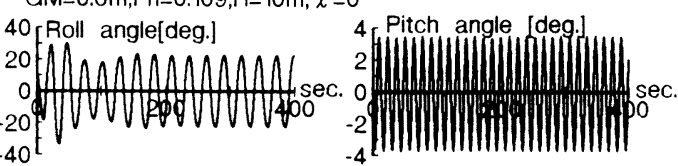

Fig. 5 Time histories of roll and pitch in wave height near critical mode leading to capsize in parametric resonance

rolling angle is 10 degrees to starboard. The pitch record may be used to judge the number of wave encounters. In these examples the rolling occurs at one half of the wave encounter frequency. In addition when the ship has a small heading angle $\chi=15$ degrees at the 
same encounter frequency as $\omega_{e}=2 \omega_{\phi}$, the critical rolling occurs at the wave encounter frequency as shown in Fig. 6 for $G M=0.318 \mathrm{~m}$ and $0.6 \mathrm{~m}$. When the ship is travelling without heading angle, the ship will roll at the natural rolling frequency induced by the $G Z$-variation in a longitudinal wave. But when the ship is travelling with heading angle, the ship will roll at the encounter frequency induced by wave excitation. That may be the reason why the rolling frequency is different. The critical wave height leading to capsize of parametric and harmonic resonance in regular waves is shown in Fig. 7. For parametric resonance the minimum wave height to cause capsize is about $4 \mathrm{~m}$ at $\chi=$ $60 \mathrm{deg}$. for $\mathrm{GM}=0.318 \mathrm{~m}$ and about $10 \mathrm{~m}$ at $\chi=45 \mathrm{deg}$. for $\mathrm{GM}=0.6 \mathrm{~m}$.

Secondly the harmonic resonances at $\omega_{e}=\omega_{\phi}$ are simulated for the ship cruising at $F_{n}=0.2745$ with $\mathrm{GM}=$ $0.318 \mathrm{~m}$ and $\mathrm{F}_{\mathrm{n}}=0.2547$ with $\mathrm{GM}=0.6 \mathrm{~m}$ and heading angle $\chi=0$ in regular wave of $\lambda / L=1$. The time histories of roll and pitch in waves of height near critical modes leading to capsize as shown in Fig. 8. The initial

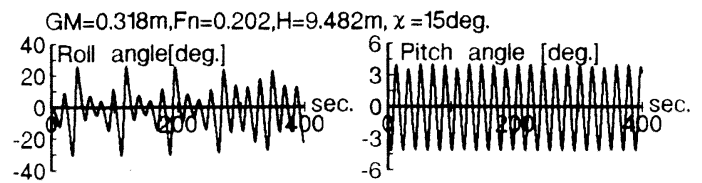

$\mathrm{GM}=0.6 \mathrm{~m}, \mathrm{Fn}=0.113, \mathrm{H}=13.22 \mathrm{~m}, \chi=15 \mathrm{deg}$

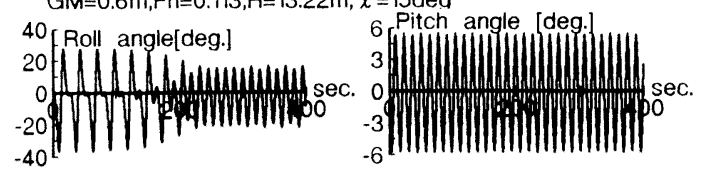

Fig. 6 Time histories of roll and pitch in wave height near critical mode leading to capsize in parametric resonance

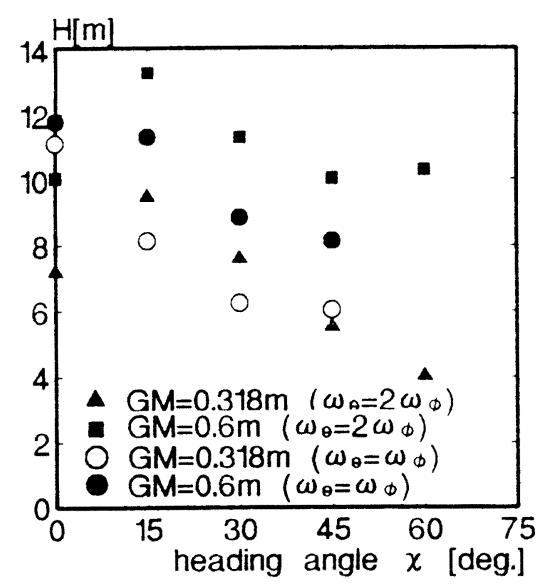

Fig. 7 Critical wave height leading to capsize of parametric and harmonic resonance in regular waves
$\mathrm{H}[\mathrm{ml}$

$\mathrm{GM}=0.318 \mathrm{~m}, \mathrm{Fn}=0.2745, \mathrm{H}=11.05 \mathrm{~m}, \chi=0$
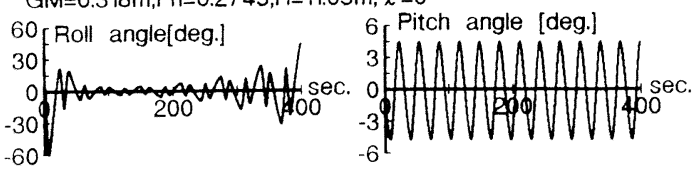

$\mathrm{GM}=0.6 \mathrm{~m}, \mathrm{Fn}=0.2547, \mathrm{H}=11.7 \mathrm{~m}, \chi=0$
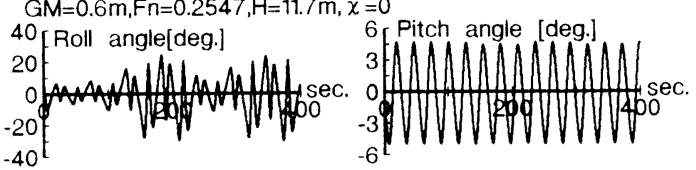

Fig. 8 Time histories of roll and pitch in wave height near critical mode leading to capsize in harmonic resonance

roll angle is 5 degrees to starboard. In these case the rolling occurs at the same wave encounter frequency. In addition when the ship with small heading angle $\chi=$ $15 \mathrm{deg}$. at the same encounter frequency as $\omega_{e}=\omega_{\phi}$, the critical rolling experience at the wave encounter frequency as shown in Fig. 9 for $\mathrm{GM}=0.318 \mathrm{~m}$ and $0.6 \mathrm{~m}$. The increment of small wave height will cause the ship immediately capsize. As mentioned above when the ship is travelling without heading angle, she will roll at the natural roll frequency, otherwise the ship with heading angle, she will roll at the encounter frequency

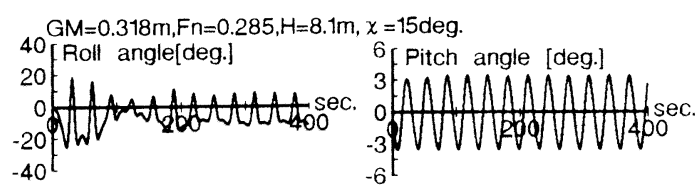

$\mathrm{GM}=0.6 \mathrm{~m}, \mathrm{Fn}=0.2635, \mathrm{H}=11.289 \mathrm{~m}, \chi=15 \mathrm{deg}$

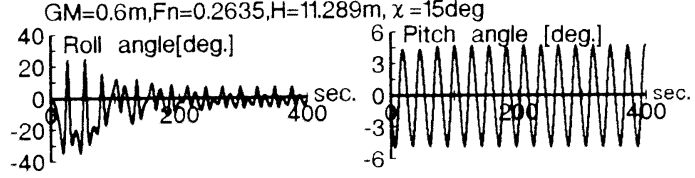

Fig. 9 Time histories of roll and pitch in wave height near critical mode leading to capsize in harmonic resonance

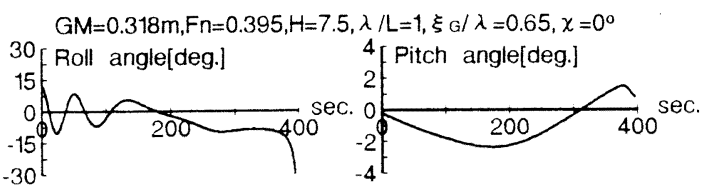

$\mathrm{GM}=0.6 \mathrm{~m}, \mathrm{Fn}=0.395, \mathrm{H}=11.06 \mathrm{~m}, \lambda / L=1, \xi_{\mathrm{G}} / \lambda=0.65, \chi=0^{\circ}$

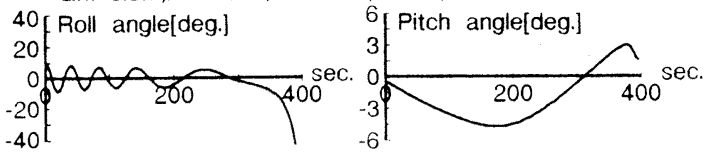

Fig. 10 Time histories of roll and pitch in wave height near critical mode leading to capsize in pure loss of stability 
induced by wave excitation. This is because the rolling frequency equal to wave encounter frequency. The critical wave height leading to capsize due to harmonic resonance in regular waves is shown in Fig. 7. The minimum wave height to cause capsize is about $6 \mathrm{~m}$ at $\chi=45 \mathrm{deg}$. for $\mathrm{GM}=0.318 \mathrm{~m}$ and about $8 \mathrm{~m}$ at $\chi=45 \mathrm{deg}$. for $\mathrm{GM}=0.6 \mathrm{~m}$.

Thirdly pure loss of stability at encounter frequency nearly equal to zero are simulated for the ship running at $\mathrm{Fn}=0.395$ and heading angle $\chi=0$ in regular wave of $\lambda / L=1$ for the cases of $\mathrm{GM}=0.318 \mathrm{~m}$ and $0.6 \mathrm{~m}$. The time histories of roll and pitch in critical wave height leading to capsize is presented in Fig. 10. It is observed that the ship is rolling with natural rolling period before capsize at wave crest amidships. This would be a typical mode of capsize due to pure loss of stability.

Probability of capsizing in random waves

The same method as that used in regular waves can be available to find out the critical rolling leading to capsize of the ship running in random waves. The probability of capsize can be obtained from numerical simulation at the critical rolling of parametric and harmonic resonance. Specifying the average period $T_{01}$ and significant wave height $H_{1 / 3}$ of wave spectrum $S(\omega)$, it is possible to obtain the time histories of random waves. The encounter frequency of a random sea is defined by average period $T_{01}$ corresponding to period of regular waves to specify $F n \cos \chi$ by making use of Fig. 1.

First examples are the time histories of random wave profile and pitch at critical rolling of parametric resonance which is shown in Fig. 11 for $\mathrm{GM}=0.318 \mathrm{~m}$ and in Fig. 12 for $\mathrm{GM}=0.6 \mathrm{~m}$, respectively. Second examples are the time histories of random wave profile and pitch at critical rolling of harmonic resonance shown in Fig. 13 for $G M=0.318 \mathrm{~m}$ and in Fig. 14 for $\mathrm{GM}=0.6 \mathrm{~m}$, respectively. Third examples are the time histories of wave profile, roll and pitch at critical rolling of pure

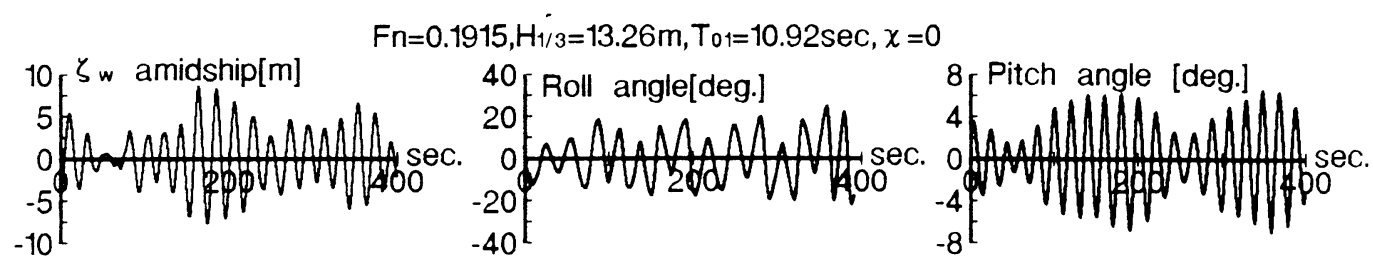

$\mathrm{Fn}=0.221, \mathrm{H}_{1 / 3}=8 \mathrm{~m}, \mathrm{~T}_{01}=10.92 \mathrm{sec}$., $\chi=30^{\circ}$

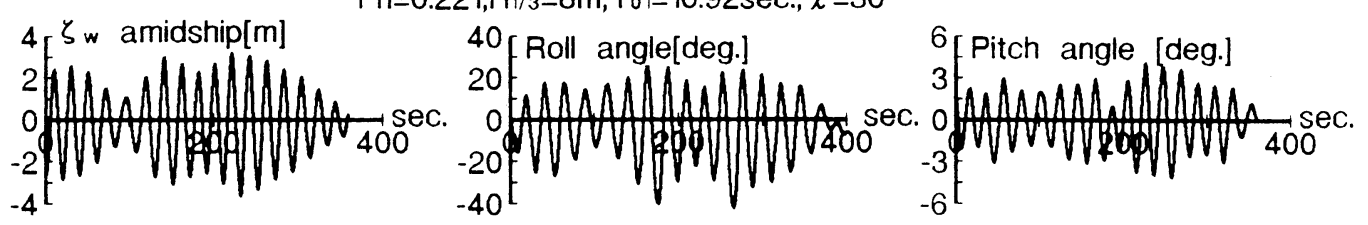

Fig. 11 Time histories of wave profile, roll and pitch angle of container at critical condition of parametric resonance for $\mathrm{GM}=0.318 \mathrm{~m}$

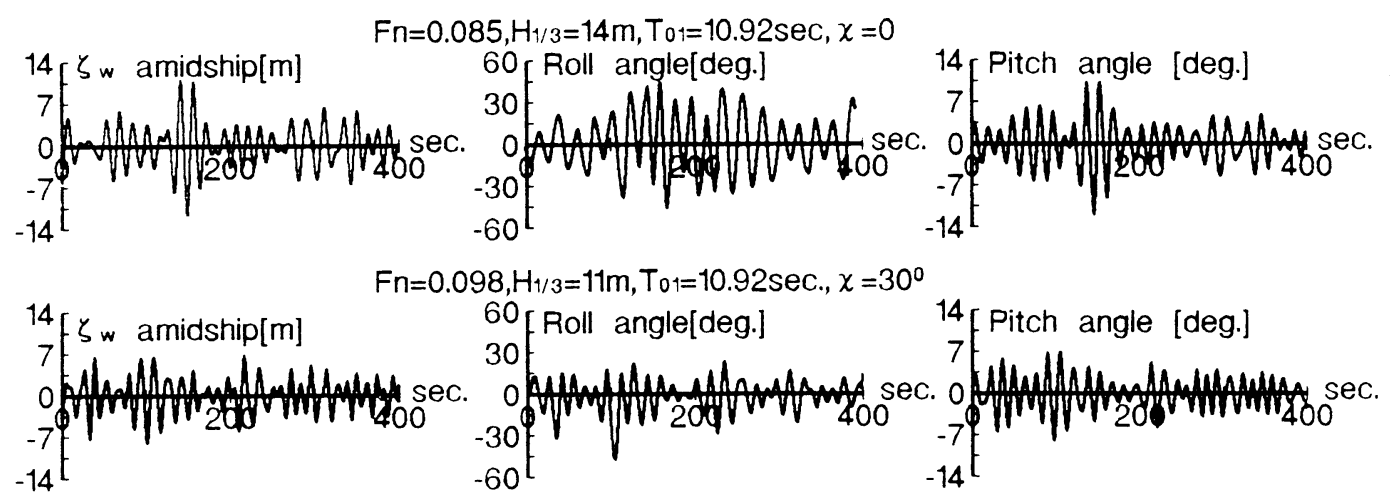

Fig. 12 Time histories of wave profile, roll and pitch angle of container at critical condition of parametric resonance with $\mathrm{GM}=0.6 \mathrm{~m}$ 
$\mathrm{Fn}=0.318, \mathrm{H}_{1 / 3}=12 \mathrm{~m}, \mathrm{~T}_{01}=10.92 \mathrm{sec}, \quad \chi=0$
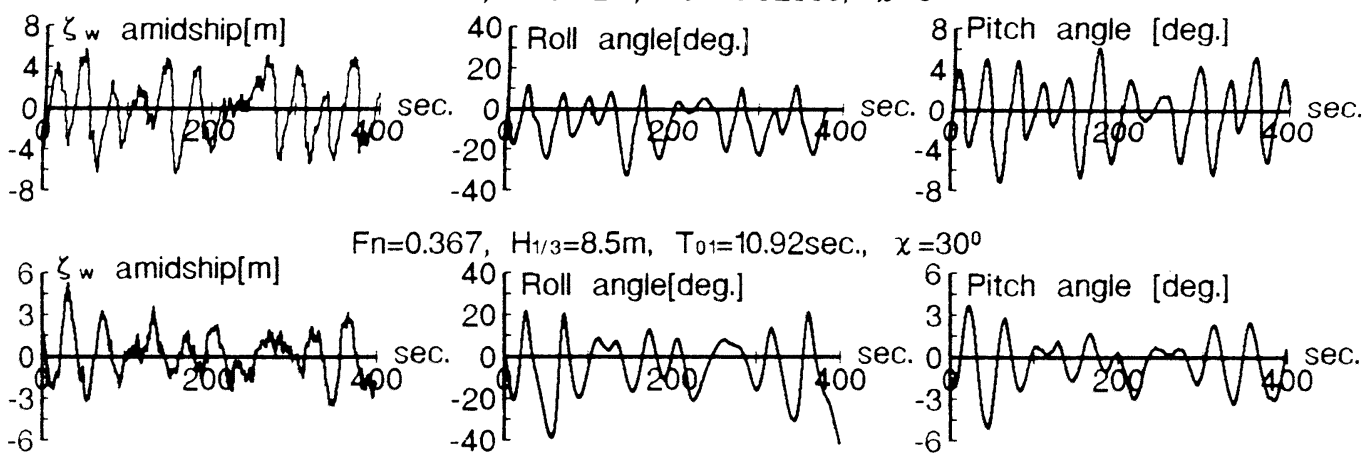

Fig. 13 Time histories of wave profile, roll and pitch angle of container at critical condition of harmonic resonance for $\mathrm{GM}=0.318 \mathrm{~m}$
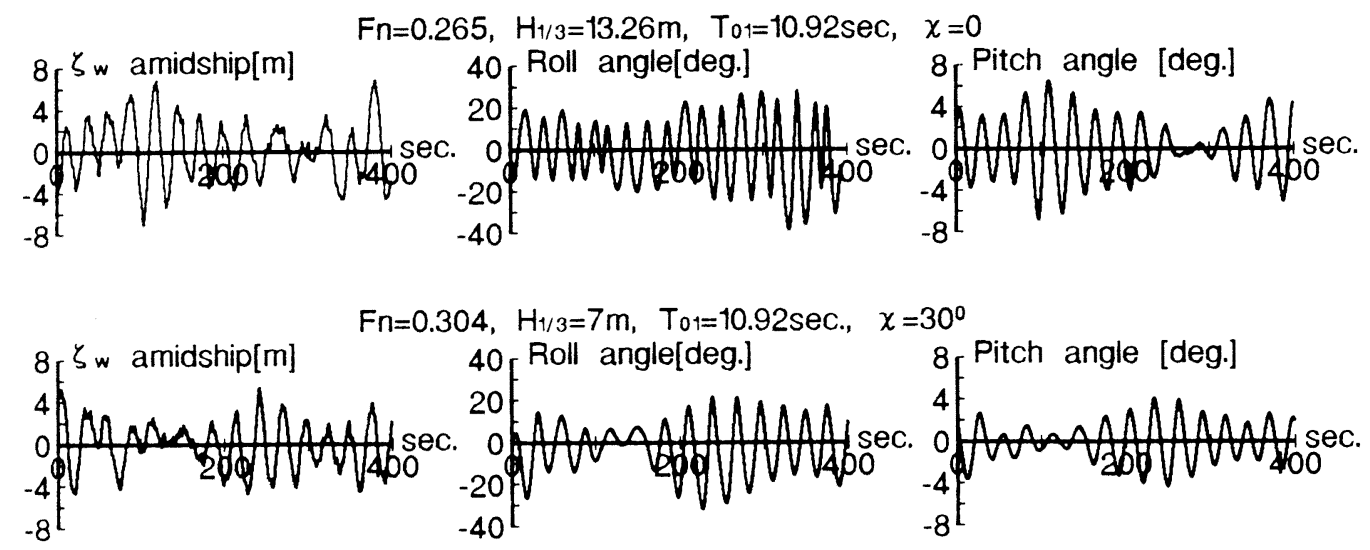

Fig. 14 Time histories of wave profile, roll and pitch angle of container at critical condition of harmonic resonance for $\mathrm{GM}=0.6 \mathrm{~m}$

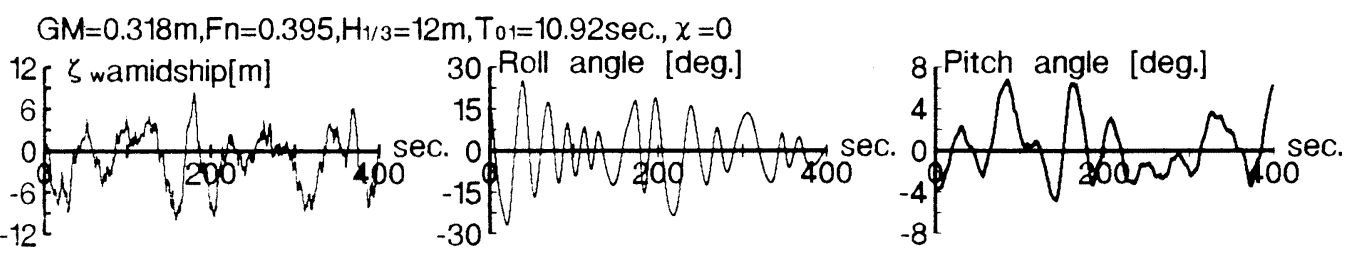

$\mathrm{GM}=0.6 \mathrm{~m}, \mathrm{Fn}=0.395, \mathrm{H}_{1 / 3}=13.26 \mathrm{~m}, \mathrm{~T}_{01}=10.92 \mathrm{sec}$. $\chi=0$

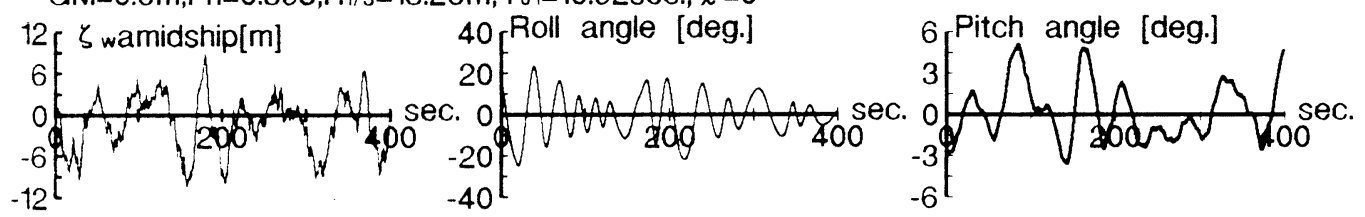

Fig. 15 Time histories of wave profile, roll and pitch angle of container at critical condition of pure loss of stability with $\mathrm{GM}=0.318 \mathrm{~m}, 0.6 \mathrm{~m}$ 
loss of stability which is shown in Fig. 15 for the heading angle $\chi=0$, and $\mathrm{GM}=0.318 \mathrm{~mm}, 0.6 \mathrm{~m}$.

The probability of up-crossing $P\left(\phi>\phi_{0}\right)$ and probability of coefficient $\mathrm{A}, P(A>0)$ can be obtained from the time histories of the critical rolling. Since the coefficient $A$ varies with the control point $\phi_{0}$, both of $P(\phi>0)$ and $P(A>0)$ also vary with the control point $\phi_{0}$ as shown in Fig. 16.

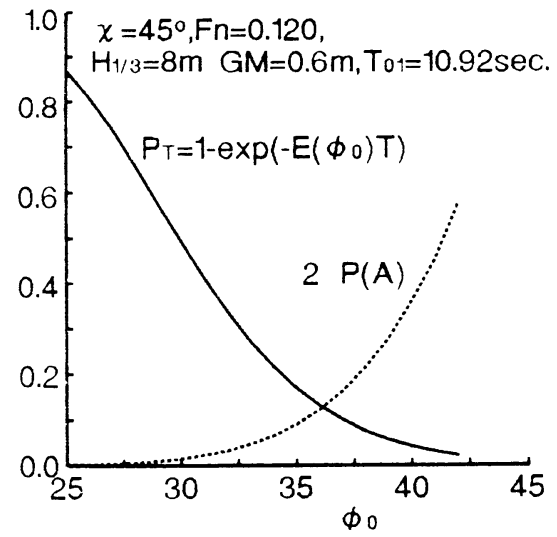

Fig. 16 Probability of up-crossing and probability of positivity of coefficient A for various control point $\phi_{0}$
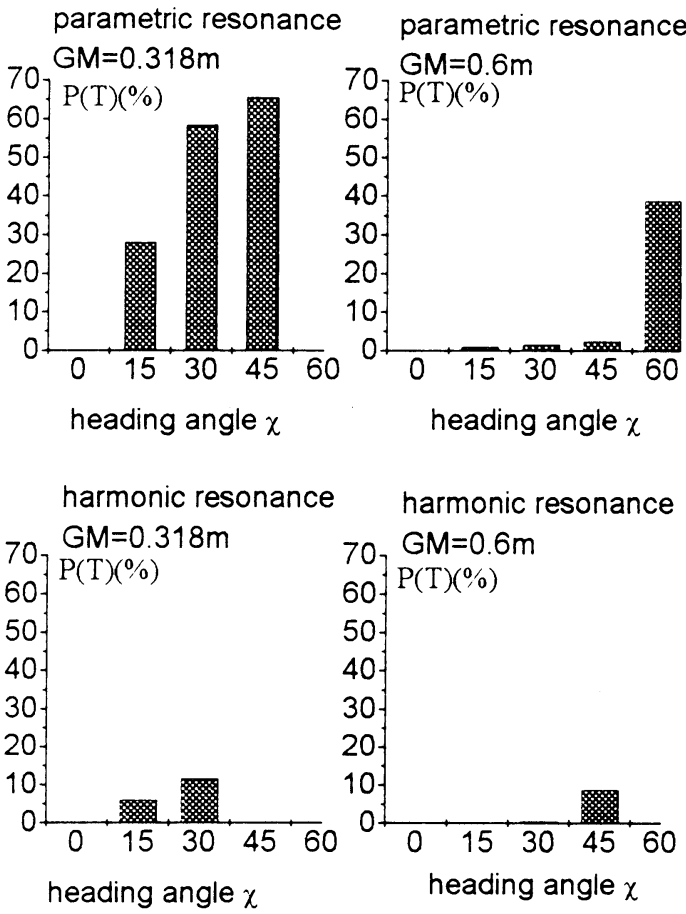

Fig. 17 Maximum Probability of capsizing during given time $\mathrm{T}$ in various heading angle $\chi$
On the other hand, the variances of roll angle $\sigma_{\phi}^{2}$ and roll velocity $\sigma_{\dot{\phi}}^{2}$ are obtained from the time histories of numerical simulation by making use of Eq. ( 7 ). Finally it is possible to obtained the maximum probability of capsizing during time $T$ as shown in Fig. 17. The probability of capsizing in parametric resonance during the given time $T$ is in the highest occurence at the heading angle $\chi=45 \mathrm{deg}$. for $\mathrm{GM}=0.318 \mathrm{~m}$ and heading angle $\chi=60 \mathrm{deg}$. for $\mathrm{GM}=0.6 \mathrm{~m}$. The probability of capsizing in harmonic resonance during the given time $T$ is in the highest occurence at the heading angle $\chi=$ $30 \mathrm{deg}$. for $\mathrm{GM}=0.318 \mathrm{~m}$ and heading angle $\chi=45 \mathrm{deg}$. for $\mathrm{GM}=0.6 \mathrm{~m}$. Since the vanishing stability angle remarkbly vary with the relative position of ship to waves when the ship is running with the heading angle $\chi=0$. This effect is not taken into account for probability computation in Fig. 17, That may be the reason why the capsizing probability is very small.

\section{Concluding Remarks}

The critical wave height leading to capsize and the probability of capsize in a random sea are investigated by making use of mathematical model for numerical simulation of ship rolling motion and a simplified method for capsizing probability. The main conclusions are summarized as follows

(1) The ship has a tendency to capsize in quartering seas more than in following seas, because the ship rolling in quartering seas is affected by both of wave induced stability and wave excitation.

(2) The parametric resonance in a lower encounter frequency range is more dangerous than the harmonic resonance in a higher encounter frequency range because the rolling motion at parametric resonance is directly developed from periodic wave induced stability related to the first Mathieu unstable frequency.

( 3 ) The probability of capsize in a random sea is in a higher occurence when the ship is running in quartering sea, this tendency is the same as results of free running model experiment.

(4) It is still now difficult to take into account the effect of wave induced stability on the probability of capsize because the control point $\phi_{0}$ changes with the relative position of ship to waves. This will be a problem to be developed in the future.

\section{References}

1) Grim, O.: Rollschwingungen, Stabilität und Sicherheit im Seegang, Schiffstechnik, Vol. 1, (1952), pp. 10-21.

2) Kerwin, J. E. : Notes on Rolling in Longitudinal Waves, International Shipbuilding Progress, Vol. 2, No. 16, (1955), pp. 597-614.

3) Paulling, J. R.: The Transverse Stability of a Ship in a Longitudinal Seaway, Journal of Ship Research, SNAME, Vol. 4, No. 4, (1961), pp. 3749.

4) Belenky, V., : A Capsizing Probability Computa- 
tion Method, Journal of Ship Research, Vol. 37, No. 3, SNAME, (1993), pp. 200-207.

5) Belenky, V., : Piece-wise Linear Methods for the Probabilistics Stability Assessment for Ship in a Seaway, STABILITY' 94, Vol. 5, (1994).

6) Hamamoto, M., Fujino, M., : Capsizes of Ship in Following Seas, Third Symposium on Marine Dynamics, The Society of Naval Architect of Japan, (1986), pp. 125-157.

7) IMO,: The IMO Intact Stability Criteria, Resolution A. 562, (1985).

8) Hamamoto, M., Sera, W., Panjaitan, J. P, : Analyses on Low Cycle Resonance of Ship in Irregular Astern Seas, Journal of the Society of Naval Architects of Japan, Vol. 178, (1995), pp. 137-145.

9) Umeda N., Hamamoto, M., Takaishi Y., Chiba Y., Matsuda A., Sera, W., Suzuki S., Spyrou K., Watanabe K., : Model Experiment of Ship Capsize in Astern Seas, Journal of the Society of Naval Architect of Japan, (1995), Vol. 177, pp. 207-217.

10) Hamamoto, M., Enomoto, T., Sera, W., Panjaitan, J. P., Ito, H., Takaishi, Y., Kan, M., Haraguchi, T., Fujiwara, T., : Model Experiment of Ship Capsize in Astern Seas (Second Report), Journal of the Society of Naval Architect of-
Japan, (1996), Vol. 179, pp. 77-87.

11) Grochowalski, S., : Investigation into the Physics of Ship Capsizing by Combined Captive and FreeRunning Model Test, SNAME Transaction, 97, (1989), pp. 169-212.

12) Tsuchiya, T., Kawashima, R., Takaishi, Y., and Yamakoshi, Y., : Capsizing Experiments of Fishing Vessesls in Heavy Seas, Proceedings of International Symposium on Practical Design inShipbuilding, Tokyo, (1977), pp. 287-294.

13) Paulling, J. R., O. H. Oakley and P. D. Wood: Ship Capsizing in Heavy Seas: The Correlation of THeory and Experiments, Proceedings of the International Conference on Stability of Ships and Ocean Vehicles, Glasgow, (1975).

14) Yamakoshi, Y., Takaishi, Y., and Kan, M., : Model Experiments on Capsize of Fishing Boats in Waves, Proceedings on the 2-nd International Conference on Stability of Ships and Ocean Vehicles, Tokyo, (1982), pp. 199-214.

15) Kan, M., Saruta, T., and Taguchi, H., Yasuno, M., and Takaishi Y., : Model Test on Capsizing of Ships in Quartering Waves, Proceedings on the 4-th International Conference on Stability of Ships and Ocean Vehicles, Naples, (1990), pp. 109116. 